\title{
Giant Ovarian Mucinous Cystadenoma Complicating Term Pregnancy
}

\author{
Sangeeta Mishra, ${ }^{1}$ Manoj Yadav, ${ }^{1}$ Sripad J Walawakar ${ }^{2}$ \\ 'Department of Obstetrics and Gynecology, Koshi Zonal Hospital, Nepal, ${ }^{2}$ Department of Pathology, Birat Medical \\ College and Teaching Hospital, Nepal.
}

\section{ABSTRACT}

Huge ovarian cyst are found in less than $1 \%$ of all ovarian cyst in pregnancy and are associated with poor feto-maternal outcome. A 28 years old G2P1 with history of normal vaginal delivery 3 years back was referred from local health post with complains of intermittent pain abdomen at 29 weeks. Her scan showed huge ovarian cyst of $18.9 \times 17.6 \mathrm{~cm}$ with multiple thick septation. Woman was conservatively managed till term and elective surgery was planned however she presented in labour with breech presentation at 39 weeks and 4days. Emergency lower segment caesarian section along with left sided salpingo-oophorectomy was done along with delivery of $2.5 \mathrm{~kg}$ healthy female baby. Histopathology was suggestive of mucinous cystadenoma of ovary. Although antepartum removal of ovarian cyst has been recommended to ensure good pregnancy outcome, expectant management and timed intervention can be adopted for pregnancy with huge ovarian cysts.

Keywords: mucinous cystadenoma; ovarian cyst; pregnancy.

\section{INTRODUCTION}

Detection of ovarian cyst during pregnancy has become more common due to routine use of ultrasonography in antenatal care. The reported incidence of pelvic mass varies from 0.5 to 2.2 percent. $^{1}$ Out of this occurrence of giant ovarian tumour is less than $1 \%$ of all the ovarian cyst during pregnancy. ${ }^{2}$ About $60 \%$ of total adnexal mass during pregnancy constitute of dermoid and mucinous cyst. Huge cyst increases maternal morbidity due to abdominal pain, torsion, rupture and secondary infection of the cyst. It may cause wrong calculation of gestational age, malpresentation, obstructed labour and may affect fetal growth as well as can cause IUGR. No specific protocol has been developed till date hence the case management varies from case to case. Main stay of management include tumour size, type, origin, gestational age, presenting symptoms and medical condition of these women. ${ }^{3}$ The present case report describes a rare occurrence of huge ovarian cyst in term pregnancy associated with malpresentation and its successful management.

\section{CASE REPORT}

A 28 year old woman was referred from a local health post with history of amenorrhea for 7 months and history of intermittent pain abdomen. The woman was G2P1 with history of normal vaginal delivery 3 years back. She was asymptomatic till now and had taken routine supplementation of iron and calcium along with two doses of tetanus toxoid from health post. On calculation with her LMP she was 29 weeks 5 days of gestation. She had not done any blood investigation

Correspondence: Dr. Sangeeta Mishra, Department of Obstetrics and Gynecology, Koshi Zonal Hospital, Nepal. Email: drsangeetamishra@gmail.com, Phone: +977-9852021741. 
however she had an ultrasound scan done at 17 weeks which was suggestive of large abdomino pelvic mass of $13.1 \times 13.3 \mathrm{~cm}$ extending to left hypochondrium and left lumber region and pushing the uterus towards right side. The finding of ovarian cyst with pregnancy was overlooked at the local health post. She was not counseled or advised anything specifically for the mass hence she never consulted any specialist but kept on taking the regular supplementation of iron and calcium tablets provided by them. She was asymptomatic till 27 weeks and developed intermittent pain abdomen of varying severity. She was referred to hospital due to same complain from the health post.
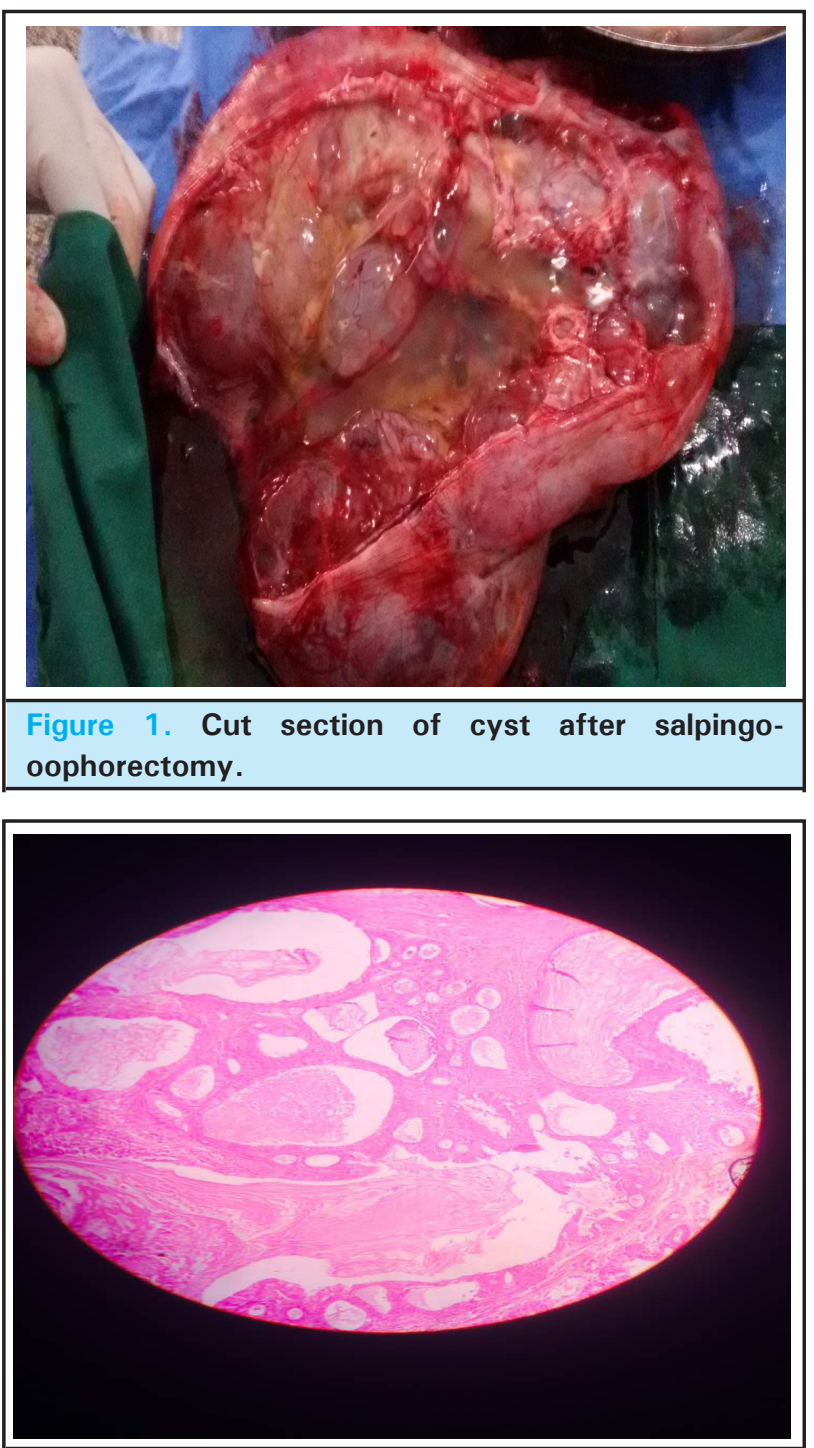

Figure 2. Low magnification picture of cyst showing multiple cystic areas showing tall columnar epithelium and stroma suggestive of mucinous cystadenoma.

On examination her vital signs were stable, per abdominal examination suggested fundal height of almost 34 weeks with regular fetal heart. Abdomen seemed tense and vague tenderness was present. There was no bleeding or discharge and pervaginal examination suggested closed cervix. All routine blood investigations done and were within normal range. Ultrasonography showed a single live fetus of 27 weeks with a large multi sepatated multicystic abdominopelvic mass in left side extending superiorly above umbilicus measuring $18.9 \times 17.6 \mathrm{~cm}$ having thick internal septation showing presence of internal vascularity and multiple tiny internal echoes. The lesion causing marked mass effect on gravid uterus displacing it to the right side and on urinary bladder.

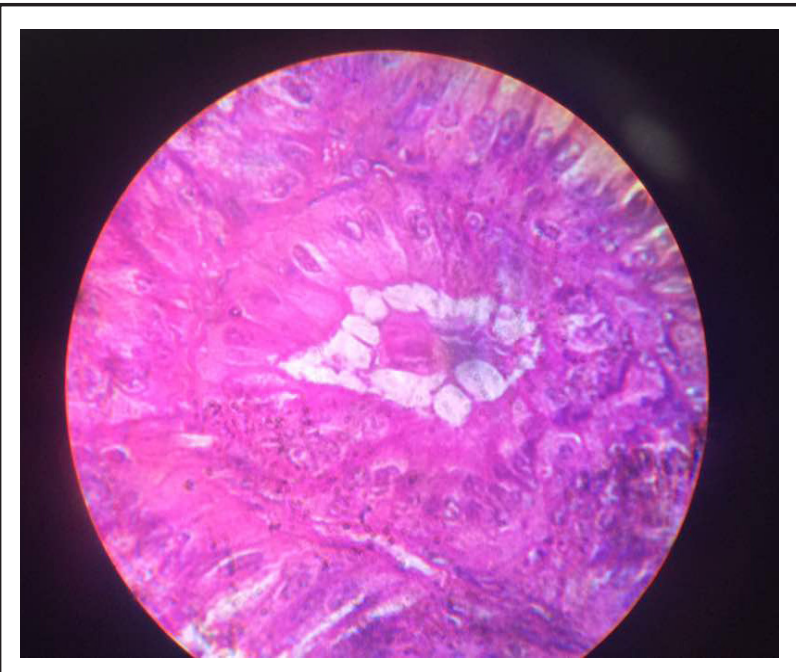

Figure 3. High magnification of cyst showing tall columunar, non ciliated cells, basal nuclei and abundant intracellular mucin.

The women and her relatives were counseled about the condition and all the management options were discussed. After discussion conservative management approach was adopted and she was kept on regular follow up. She was given medications for pain relief and was advised to report immediately if pain increased or any complication occurred. She came for regular follow up till 34 weeks and was planned for an elective Caesarian section at 38 completed weeks. However she did not turned up at 38 weeks and presented to emergency at 39 weeks and 4 days of gestation with history of intermittent labour pain. On examination her vital signs were stable with pulse 82/ min and BP 130/80. Abdominal examination revealed an over distended uterus with regular contraction of 30 seconds every two minutes with regular fetal heart sound. Per vaginal examination suggested $3 \mathrm{~cm}$ dilated and $50 \%$ effaced cervix with buttocks as presenting part. Provisional diagnosis of G2P1 at 39 weeks 4 days of gestation in latent stage of labour with breech presentation with huge ovarian cyst was made and the woman was taken up for emergency caesarian section under spinal anesthesia. She delivered a female baby 
Mishra et al. Giant Ovarian Mucinous Cystadenoma Complicating term pregnancy

of $2.5 \mathrm{~kg}$ weight with an APGAR 6,8,10. Left sided salpingo oophorectomy was done at the same sitting. There was a huge left sided ovarian tumor of $25 \times$ $19 \mathrm{~cm}$ and weighed $3.3 \mathrm{Kg}$. Ovarian mass was sent for histopathological examination. Woman recovered fully and was discharged on $7^{\text {th }}$ post operative day after suture removal. The histopathology of mass was suggestive of benign mutinous cyst adenoma of ovary.

\section{DISCUSSION}

Most of the ovarian masses are asymptomatic and are diagnosed incidentally during pregnancy due to routine use of ultrasound. Cysts more than 3 centimeters are detectable on sonography and usually decline spontaneously after 10 weeks of gestation. Lesions persisting beyond 12 weeks associated with complex sonological features are neoplasm and are usually benign. ${ }^{4,5}$ The most common symptom of ovarian masses is pain which may be mild to severe if torsion or rupture takes place and warrants immediate surgery. ${ }^{3}$ Normal physiological and anatomical changes during pregnancy may cause some pain so the pathological conditions like ovarian tumor gets ignored in absence of routine scan as in the present case. The mainstay of diagnosis is ultrasound which is $96.8 \%$ sensitive and $77 \%$ specific for diagnosing ovarian masses however MRI can be suggested in equivocal cases if available. ${ }^{5,6}$ Advantage of MRI over sonography is that it can visualize the more posterior and lateral area of the pelvis which may be obscured by fetal bones. It is safe as it does not involve harmful ionizing radiation and has accuracy rate of $100 \% .^{7}$ The role of tumor makers in pregnancy is equivocal as all the markers CA 125, CEA, alpha feto protein are usually elevated during pregnancy and varies in each trimester so are difficult to conclude if used alone and without imaging modalities. ${ }^{8}$

Management is primarily surgical. Tumor size, gestational age and primary presenting complain are the most important factors affecting the surgical removal. The risk of spontaneous abortion in first trimester and preterm labour and IUGR in third trimester should always be kept in mind. ${ }^{9}$ Second trimester is the best time for surgery for the removal of ovarian masses as the risk is low compared to first and third trimester. Laparoscopic management of the cyst may be considered due to the advantage of short hospital stay and lesser blood loss however this approach requires expertise and may not be possible in women with large tumors and with advanced gestation. ${ }^{10}$ Considering watchful management and reserving surgery in case of onset of symptoms and emergencies can be considered. There are many case reports that considered watchful expectancy as the best management approach for huge ovarian cyst presenting in third trimester and removed the cyst at the time of elective caesarian section. ${ }^{11-12}$ Qublan et al described a $6300 \mathrm{gm}$ of multiloculated right ovarian cystadenoma measuring $33 \times 24 \times 20 \mathrm{~cm}$ at 38 weeks of pregnancy associated with IUGR and malpresentation. This was similar in our case as this woman had a breech presentation and low birth weight of $2.5 \mathrm{~kg}$. To the best of our knowledge this is the first case from Nepal of a huge mucinous cystadenoma associated with term pregnancy malpresentation and low birth weight.

In our case we discussed all the possibilities and complication of this huge tumor on pregnancy and immediate removal. Expectant management was chosen and woman was carefully monitored and elective surgery was planned at 38 completed weeks. However this woman could not come at 38 weeks and presented at 39 weeks 4 days in labour an emergency lower segment caesarian section along with left sided salphingo oophorectomy was done with optimal fetomaternal outcome.

\section{Conflict Of Interest: None.}

Consent: JNMA Case Report Consent Form was signed by the patient and the original article is attached with the patients chart.

\section{REFERNCES}

1. Hill L M, Connors-Beatty DJ, Nowak Tush BA. The role of ultrasonography in detection and management of adnexal masses during the second and third trimesters of pregnancy. Am J obstet Gynecol. 1998;179:703-7. [Full Text]

2. Boulay R, Podczaski E. Ovarian Cancer Complicating Pregnancy. Obstet Gynae4col Clin North Am. 1998;25(2):385-99. [Full Text]

3. Leiserowitz GS. Managing ovarian Masses during pregnancy. Obstet Gynecol Surv. 2006;61(7): 463-70. [PubMed|DOI]
4. Elhalwagy H. Management of ovarian masses in pregnancy. Trends Urol Gynecol Sex Health. 2009;14(1):14-8. [E Full Text]

5. Ross EK, Kebria M. Incidental Ovarian cysts: when to reassure, when to reassess, when to refer. Cleve Clin J Med. 2013:80(8):503-14. [PubMed $\mid \underline{\text { PMC] }}$ 
6. Batool K, Reza JE, Jila A. Papillary serous cystadenoma of ovary: A huge ovarian cyst complicating the pregnancy. J Cases Obstet Gynecol. 2016;3(4):121-4. [Full Text]

7. Curtis M, Hopkins MP, Zarlingo T, Martino C, Graciansky-Lengyl M, Jenison EL., Magnetic resonance imaging to avoid laparotomy in pregnancy. Obstet Gynaecol. 1993; 829(5): 833-6. [PubMed | PMC]

8. Ercan Ş, Kaymaz Ö, Yücel N, Orçun A. Serum concentration of CA 125, Ca 15-3, CA 19-9 and CEA in normal pregnancy: a longitudinal study. Arch Gynecol Obstet. 2012 Mar 1;285(3):579-84. [ PubMed | DOI]

9. Mazze R I, Kallen B. Reproductive outcome after anesthesia and operation during pregnancy: a registry study of 5405 cases. Am J Obstet Gynaecol. 1989;16(5): 1178-85. [PubMed I $\underline{\mathrm{PMC}}]$
10. Cavaco - Gomes J, Jorge Moreira C, Rocha A, Mota R, Paiva $\mathrm{V}$, Costa A. Investigation and Management of Adnexal Masses in Pregnancy. Scientifica (Cairo). 2016;2016:3012802 [PubMed | Full Text]

11. Qublan HS, AL- ghoweri AS, Al-Kaisi NS, Abu-Khait SA. Benign mucinous cystadenoma with stromal leutinization during pregnancy: a hormonally responsive tumour and a rare cause of fetal intrauterine growth retardation.J obstet Gynaecol Res. 2002 Apr;28(2):104-7. [PubMed | PMC]

12. Gonca IY, Meral C, Sema A. A huge ovarian cystadenoma complicating pregnancy: A case report. Cumhuriyet Med J. 2009;31:174-17. [Full Text] 